\title{
NPR-C gene polymorphism is associated with increased susceptibility to coronary artery disease in Chinese Han population: a multicenter study
}

Qin Hu ${ }^{1}$, Qiji Liu ${ }^{2}$, Shasha Wang ${ }^{1}$, Xi Zhen ${ }^{1}$, Zhimian Zhang ${ }^{3}$, Ruijuan Lv ${ }^{4}$, Guihua

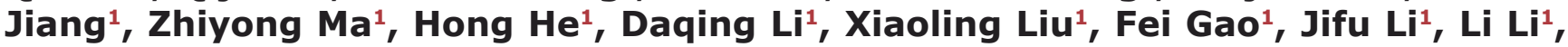
Mei Zhang ${ }^{1}$, Xiaoping $\mathrm{Ji}^{1}$, Yuguo Chen ${ }^{4}$, Daowen Wang ${ }^{5}$, Dejia Huang ${ }^{6}$, Aiqun Ma ${ }^{7}$, Wei Huang ${ }^{8}$, Yuxia Zhao', Yaoqin Gong ${ }^{2}$, Cheng Zhang ${ }^{1}$ and Yun Zhang ${ }^{1}$

${ }^{1}$ Key Laboratory of Cardiovascular Remodeling and Function Research, Chinese Ministry of Education and Chinese Ministry of Health, Department of Cardiology, Shandong University Qilu Hospital, Jinan, Shandong, China

2 Department of Medical Genetics, School of Medicine, Key Laboratory of Experimental Teratology, Ministry of Education, Shandong University, Jinan, Shandong, China

${ }^{3}$ Medical Examination Center, Shandong University Qilu Hospital, Jinan, Shandong, China

${ }^{4}$ Department of Emergency, Shandong University Qilu Hospital, Jinan, Shandong, China

${ }^{5}$ Department of Internal Medicine, Tongji Hospital, Tongji Medical College, Huazhong University of Science \& Technology, Wuhan, China

${ }^{6}$ Division of Cardiology, Department of Medicine, West China Hospital, Sichuan University, Chengdu, Sichuan, China

${ }^{7}$ Department of Cardiology and Periphery Vascular Medicine, The First Affiliated Hospital of Medical College of Xi'an Jiaotong University, Xi'an, Shaanxi, China

${ }^{8}$ Ruijin Hospital, School of Medicine, Shanghai Jiaotong University, Department of Genetics, Chinese National Human Genome Center, Shanghai, China

Correspondence to: Cheng Zhang, email: zhangc@sdu.edu.cn

Yun Zhang, email: zhangyun@sdu.edu.cn

Keywords: natriuretic peptide receptor C, coronary artery disease, genome-wide association studies, single nucleotide polymorphisms, susceptibility gene, Pathology Section

Received: November 24, $2015 \quad$ Accepted: May 04, $2016 \quad$ Published: May 13, 2016

\section{ABSTRACT}

To find a new locus that confers significant susceptibility to CAD in Chinese Han population, a genome-wide association study in $\mathbf{2 0 0}$ "extreme individuals" from a Shandong cohort and a pathway-based candidate gene study from a Shanghai cohort ( 293 CAD/293 controls) were simultaneously performed. Amongst them, 13 SNPs associated with CAD were selected to conduct validation and replication studies in additional 3363 CAD patients and 3148 controls. A novel locus rs700926 in natriuretic peptide receptor C (NPR-C) was identified in Shandong and Hubei cohorts. Then rs700926 and other nine tag SNPs were genotyped in four geographically different populations (Shandong, Shaanxi, Hubei and Sichuan cohorts), and 6 SNPs (rs700926, rs1833529, rs2270915, rs17541471, rs3792758 and rs696831) showed stronger association with CAD, regardless of single or combined analysis. We further genotyped rs2270915 and 10 additional tag SNPs in a central China cohort and identified rs12697273 and rs10066436 as the loci associated with CAD. All these positive associations remained significant after adjustment for traditional risk factors of CAD. NPR-C gene SNPs significantly contribute to CAD susceptibility in the Chinese Han population.

\section{INTRODUCTION}

Coronary artery disease (CAD) is the leading cause of mortality worldwide and more than 700,000 people die from CAD each year in China [1]. Current evidence suggests that $\mathrm{CAD}$ is a heritable trait activated 
Table 1: Characteristics of the study population

\begin{tabular}{|c|c|c|c|c|c|c|c|c|c|c|c|}
\hline \multicolumn{2}{|l|}{ Population } & \multirow{2}{*}{$\begin{array}{l}\text { Age* }^{*} \\
44.9 \pm 4.81\end{array}$} & \multirow{2}{*}{$\begin{array}{l}\begin{array}{l}\text { Gender } \\
\text { Female } \\
n(\%)\end{array} \\
20(20.0)\end{array}$} & \multirow{2}{*}{ 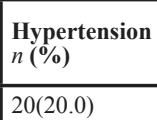 } & \multirow{2}{*}{$\begin{array}{l}\begin{array}{l}\text { Diabetes } \\
n(\%)\end{array} \\
4(4.0)\end{array}$} & \multirow{2}{*}{\begin{tabular}{|l|}
$\begin{array}{l}\text { BMI } \\
\mathbf{k g} / \mathbf{m}^{2}\end{array}$ \\
$25.1 \pm 8.5$
\end{tabular}} & \multirow{2}{*}{$\begin{array}{l}\text { Hyperlipidemia } \\
n(\%) \\
4(4.0)\end{array}$} & \multirow{2}{*}{ 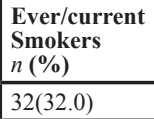 } & \multirow{2}{*}{$\begin{array}{l}\text { MI } \\
n(\%) \\
42(42.0)\end{array}$} & \multirow{2}{*}{\begin{tabular}{|l|}
$\begin{array}{l}\text { PCI } \\
n(\%)\end{array}$ \\
$56(56.0)$
\end{tabular}} & \multirow{2}{*}{$\begin{array}{l}\begin{array}{l}\text { CABG } \\
\mathbf{n}(\%)\end{array} \\
4(4.0)\end{array}$} \\
\hline \multirow{3}{*}{$\begin{array}{l}\text { Dis- } \\
\text { Shandong }\end{array}$} & Case $(n=100)$ & & & & & & & & & & \\
\hline & $\begin{array}{l}\text { Control }(n= \\
100)\end{array}$ & $62.3 \pm 6.7$ & $22(22.0)$ & $78(78.0)$ & $26(26.0)$ & NA & $32(32.0)$ & $58(58.0)$ & $0(0)$ & $0(0)$ & $0(0)$ \\
\hline & $p$ & $<0.001$ & 0.27 & $<0.001$ & $<0.001$ & $<0.001$ & $<0.001$ & $<0.001$ & $<0.001$ & $<0.001$ & $<0.001$ \\
\hline \multirow{3}{*}{$\begin{array}{l}\text { Dis- } \\
\text { Shanghai }\end{array}$} & Case $(n=293)$ & $63.3 \pm 10.5$ & 113(38.6) & 182(62.1) & $80(27.3)$ & $24.1 \pm 2.8$ & 197(67.2) & $116(39.6)$ & NA & NA & NA \\
\hline & $\begin{array}{l}\text { Control }(n= \\
293)\end{array}$ & $63.3 \pm 10.6$ & $113(38.6)$ & $197(67.2)$ & $20(6.8)$ & $23.9 \pm 3.2$ & $182(62.2)$ & $77(26.3)$ & $0(0)$ & $0(0)$ & $0(0)$ \\
\hline & $p$ & 0.541 & 0.793 & $<0.001$ & $<0.001$ & 0.282 & $<0.001$ & $<0.001$ & $N A$ & $N A$ & $N A$ \\
\hline \multirow{3}{*}{$\begin{array}{l}\text { Val- } \\
\text { Shandong }\end{array}$} & $\operatorname{Case}(n=480)$ & $59.9 \pm 9.5$ & $116(24.2)$ & $276(57.5)$ & 104(21.7) & $26.0 \pm 3.2$ & $132(27.5)$ & $258(53.8)$ & $301(62.7)$ & $257(53.5)$ & $22(4.6)$ \\
\hline & $\begin{array}{l}\text { Control }(n= \\
469)\end{array}$ & $40.8 \pm 12.8$ & 194(41.4) & $3(0.006)$ & $2(0.004)$ & $24.8 \pm 3.5$ & $1(0.002)$ & $119(25.3)$ & $0(0)$ & $0(0)$ & $0(0)$ \\
\hline & $p$ & $<0.001$ & $<0.001$ & $<0.001$ & $<0.001$ & $<0.001$ & $<0.001$ & $<0.001$ & $<0.001$ & $<0.001$ & 0.000 \\
\hline \multirow{3}{*}{$\begin{array}{l}\text { Val- } \\
\text { Hubei }\end{array}$} & Case $(n=694)$ & $59.8 \pm 11.8$ & $156(22.5)$ & $425(61.2)$ & 134(19.3) & $24.4 \pm 3.5$ & 79(11.4) & $389(56.1)$ & $319(46.0)$ & $247(35.6)$ & $9(1.3)$ \\
\hline & $\begin{array}{l}\text { Control }(n= \\
979)\end{array}$ & $49.5 \pm 10.2$ & $598(59.0)$ & $4(0.004)$ & $3(0.003)$ & $22.8 \pm 2.6$ & $1(0.001)$ & 19(1.9) & $0(0)$ & $0(0)$ & $0(0)$ \\
\hline & $p$ & $<0.001$ & $<0.001$ & $<0.001$ & $<0.001$ & $<0.001$ & $<0.001$ & $<0.001$ & $<0.001$ & $<0.001$ & $<0.001$ \\
\hline \multirow{3}{*}{$\begin{array}{l}\text { Rep- } \\
\text { Shanxi }\end{array}$} & Case $(n=392)$ & $60.1 \pm 12.3$ & $83(21.2)$ & 183(46.7) & $69(17.6)$ & $24.7 \pm 4.2$ & $16(17.4)$ & 197(50.3) & 101(25.8) & $276(70.4)$ & $7(1.8)$ \\
\hline & $\begin{array}{l}\text { Control }(n= \\
236)\end{array}$ & $40.4 \pm 18.1$ & $115(48.7)$ & $1(0.004)$ & $1(0.004)$ & $22.74 \pm 3.4$ & $1(0.004)$ & $35(14.8)$ & $0(0)$ & $0(0)$ & $0(0)$ \\
\hline & $p$ & $<0.001$ & $<0.001$ & $<0.001$ & $<0.001$ & $<0.001$ & $<0.001$ & $<0.001$ & $<0.001$ & $<0.001$ & $<0.001$ \\
\hline \multirow{4}{*}{$\begin{array}{l}\text { Rep- } \\
\text { Sichuan }\end{array}$} & Case $(n=480)$ & $65.9 \pm 10.8$ & $90(18.8)$ & $253(52.7)$ & $100(20.8)$ & $24.6 \pm 5.8$ & $67(14.0)$ & 273(56.9) & $140(29.2)$ & 464(96.7) & $8(1.7)$ \\
\hline & \begin{tabular}{|l} 
Control $(n=$ \\
$460)$
\end{tabular} & $54.1 \pm 16.1$ & $245(53.2)$ & $2(0.004)$ & $3(0.006)$ & $23.7 \pm 4.7$ & $1(0.002)$ & $55(12.0)$ & $0(0)$ & $0(0)$ & $0(0)$ \\
\hline & $p$ & $<0.001$ & $<0.001$ & $<0.001$ & $<0.001$ & $<0.001$ & $<0.001$ & $<0.001$ & 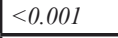 & $<0.001$ & $<0.001$ \\
\hline & Case $(n=1024)$ & $65.3 \pm 10.8$ & $272(26.6)$ & $662(64.7)$ & 182(17.8) & $24.1 \pm 2.8$ & 91(8.9) & $342(33.4)$ & NA & NA & NA \\
\hline \multirow[t]{2}{*}{$\begin{array}{l}\text { Rep- } \\
\text { Center2 }\end{array}$} & $\begin{array}{l}\text { Control }(n= \\
711)\end{array}$ & $60.2 \pm 9.0$ & $386(54.3)$ & $348(49.0)$ & $58(8.2)$ & $24.1 \pm 4.1$ & $29(4.1)$ & $151(21.2)$ & $0(0)$ & $0(0)$ & $0(0)$ \\
\hline & $p$ & $<0.001$ & $<0.001$ & $<0.001$ & $<0.001$ & $<0.001$ & $<0.001$ & $<0.001$ & NA & NA & NA \\
\hline
\end{tabular}

Age of cases and Age of controls, age at the time of enrollment. Data for age and BMI are shown as mean \pm standard deviation. We used $\chi^{2}$ tests to compare the differences between gender, hypertension, type 2 diabetes and smoking status. We used a Student's $t$ test to compare the means of the cases and controls. NA, no data; NS: no significant; BMI, body mass index; ${ }^{*}$ Age in years.

by a number of biological pathways and responsive to environmental stimuli [2] Recently, genome-wide association studies (GWAS), a technology of chip array using hundreds of thousands of markers and targeted genebased resequencing, have facilitated the gene discovery for CAD. Previous GWAS in populations of European ancestry identified several genetic loci on chromosomes $1 \mathrm{p} 13,1 \mathrm{q} 41,2 \mathrm{q} 36,3 \mathrm{q} 22,6 \mathrm{p} 24,6 \mathrm{q} 25,9 \mathrm{p} 21,10 \mathrm{q} 11,12 \mathrm{q} 24$ and $15 \mathrm{q} 22$ that are associated with risk of CAD or its major complication, myocardial infarction [3-9]. To date, few GWAS have been conducted in Chinese populations. Thus, the susceptibility genes that significantly contribute to the development of CAD in the majority of Chinese Han population remain poorly characterized.

The purpose of the present study was to find out novel susceptibility genes for CAD in Chinese Han population. Our design included a discovery study (stage 1 ), a validation study (stage 2) and a replication study (stage 3). In stage 1, we performed GWAS in "extreme individuals" from Shandong cohort and a pathwaybased candidate gene case-control association study in a Shanghai cohort in combination with the data from a British population by the Wellcome Trust Case Control Consortium (WTCCC). In stage 2, we selected 13 SNPs for validation and identified a novel susceptibility locus rs 700926 in natriuretic peptide receptor $\mathrm{C}(N P R-C)$. In stage 3, we performed case-control association studies of tag SNPs to further validate new susceptibility loci of $N P R-C$ for CAD in 6 larger cohorts from northern, southern and central China.

\section{RESULTS}

\section{Study population characteristics}

The demographical and clinical characteristics of the discovery, validation and replication populations are summarized in Table 1. The prevalence of established cardiovascular risk factors (except for gender) was significantly higher in controls than that in cases. In candidate gene association study, a lower prevalence of hypertension (62.1\% versus $67.2 \%$ ), a higher prevalence of diabetes (27.3\% versus $6.8 \%$ ) and hyperlipidemia (67.2\% versus $62.2 \%$ ), and a higher percentage of ever and /or current smoking (39.6\% versus $26.3 \%$ ) were observed in cases than in controls. In the validation and replication study populations, as anticipated, the prevalence of 
established cardiovascular risk factors was significantly higher in cases than that in controls. Overall, the patient characteristics in the present study were typical for those undergoing coronary angiography for the evaluation of CAD, with a male preponderance (over $61.4 \%$ ) and a high prevalence of diabetes (over 17.6\%), hypertension (over $46.7 \%$ ), hyperlipidemia (over $8.9 \%$ ) as well as ever and / or current smoking (over 15.4\%). Moreover, as expected, the serum TC levels were significantly higher but the serum HDL-C levels lower in cases than in controls.

\section{SNPs potentially associated with CAD}

In the discovery stage, 100 "extreme cases" and 100 "extreme controls" recruited from Shandong were genotyped using Infinium HumanOmnizhonghua-8 BeadChip (Illumina). We constructed quantile-quantile (Q-Q) plot for this population using the genome-wide genotyping data (Supplementary Figure S.1A). To ensure that case and control groups were genetically matched, in addition to close examination of their geographic origins, multidimensional scaling (MDS) was used to exclude population outliers (Supplementary Figure S.1B), the result of which was further confirmed by principalcomponent analysis (PCA), which showed minimal evidence for population stratification. A total of 8,7032 SNPs failed to achieve a call rate of $90 \%$ and thus were excluded from further analysis, whereas 78,5229 SNPs in 100 "extreme cases" and 100 "extreme controls" passed quality control and entered final statistical analysis using the Cochran-Armitage trend test to examine the genotypephenotype association under an additive model. After genomic control (GC) with an inflation factor of 1.08 [6], the association results did not change significantly. Supplementary Figure S.2 provided a plot of the metaanalysis $p$ values by chromosome position. Given a small sample size of 200 and the design of "extreme cases and controls", a $P$ value $1^{\prime} 10^{-4}$ was considered strong evidence for association in this discovery stage, although the a priori threshold for genome-wide significance was $5^{\prime} 10^{-8}$. Nonetheless, the relative small sample size in our GWAS study may result in a negative result due to limited statistical power. To avoid possible bias caused by this arbitrary selection, for SNPs with weaker associations $(P$ value between $10^{-4}$ and $10^{-3}$, Supplementary Figure S.2), we also searched signal pathway-based candidate genes related to atherosclerosis and CAD reported from previous publications. ${ }^{2}$ Combined with the reported candidate gene and the earlier reports of the highthroughput dataset from a British population by WTCCC (http://www. wtccc.org.uk/) in relation to CAD, we screened a total of 120 SNPs representing 49 genomic regions of human chromosome $1,2,3,4,5,6,7,8,9,10,11,12,13,16$, 18, 19, 20 and 22 (Supplementary Table S.1). These

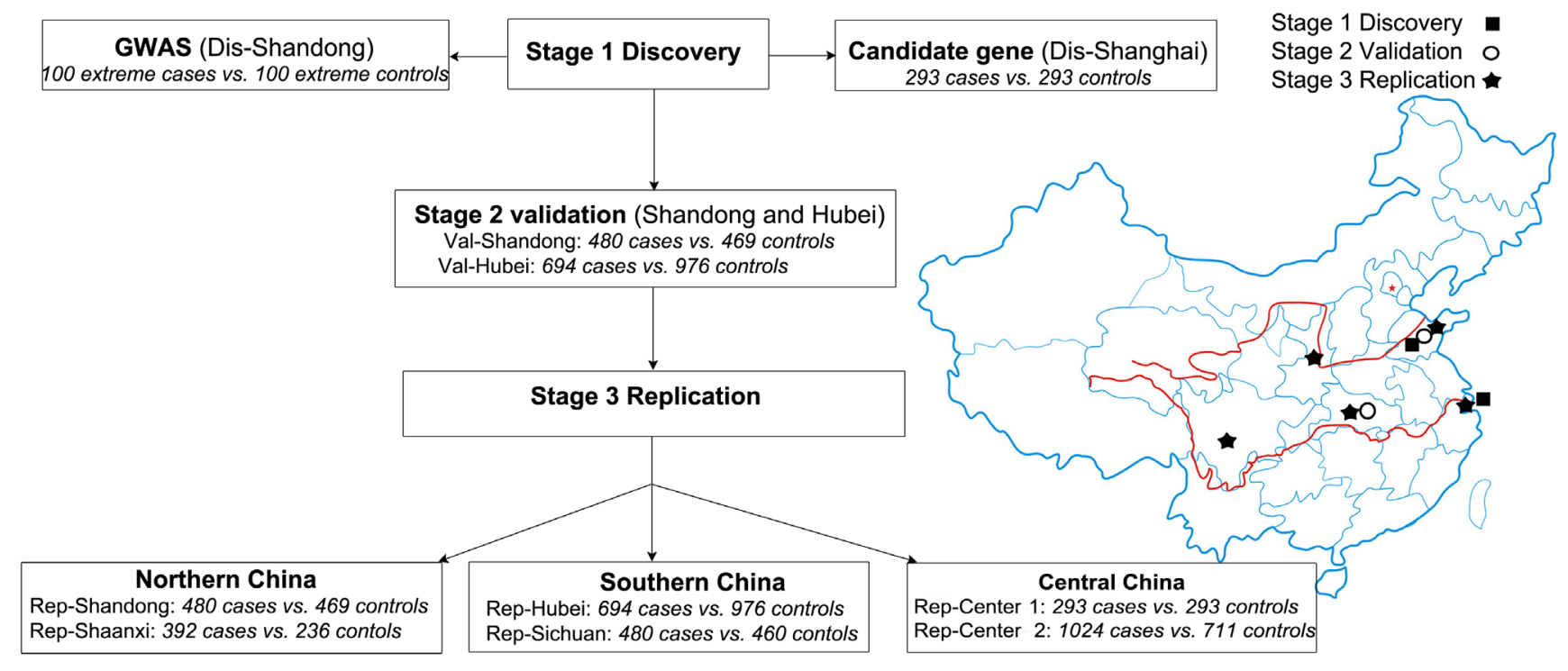

Figure 1: Flow chart of the present study. The stage 1 discovery study enrolled two cohorts of populations: a GWAS-based population in Shandong including 200 "extreme individuals" (Dis-Shandong) and a candidate gene-based study population including 596 individuals in Shanghai (Dis-Shanghai). The stage 2 validation study consisted of 480 cases and 469 controls from Shandong (Val-Shandong) and 694 cases and 979 controls from Hubie (Val-Hubei). Stage 3 replication studies of rs 700926 and the nine tag SNPs of NPR-C included 5 independent cohorts: Rep-Shanghai, Rep-Shandong, Rep-Shaanxi, Rep-Hubei and Rep-Sichuan in China. The stage 3 replication study included 480 cases and 469 controls in Shandong (Rep-Shandong), 392 cases and 236 controls in Shaanxi (Rep-Shaanxi), 694 cases and 976 controls in Hubei (Rep-Hubei), 480 cases and 460 controls in Sichuan (Rep-Sichuan), 293 cases and 293 controls in Cohort 1 in Central China (Rep-Center 1) and 1024 cases and 711 controls in Cohort 2 of Cnetral China (Rep-Center 2). Rep-Shandong is the same as ValShandong, Rep-Hubei is the same as Val-Hubei, and Rep-Center 1 is the same as Dis-Shanghai. 
Table 2: Allelic association of SNPs of $N P R-C$ with coronary artery disease in Rep-Shandong, Rep-Hubei, Re-Sichuan and Re-Shaanxi cohorts

\begin{tabular}{|c|c|c|c|c|c|c|c|c|c|c|c|c|c|c|c|c|c|}
\hline \multirow{3}{*}{ SNP_ID } & \multirow{3}{*}{$\begin{array}{l}\text { Minor } \\
\text { Allele }\end{array}$} & \multicolumn{4}{|c|}{ Re-Shandong } & \multicolumn{4}{|c|}{ Re-Hubei } & \multicolumn{4}{|c|}{ Re-Sichuani } & \multicolumn{4}{|c|}{ Re-Shaanxi } \\
\hline & & \multicolumn{2}{|c|}{ MAF(\%)* } & \multirow{2}{*}{$\begin{array}{l}\text { OR } \\
(95 \% \mathrm{CI}) \\
\dagger \\
\end{array}$} & \multirow{2}{*}{$\begin{array}{l}\text { P-obs } \\
\text { \# }\end{array}$} & \multicolumn{2}{|c|}{ MAF(\%)* } & \multirow{2}{*}{$\begin{array}{l}\text { OR } \\
(95 \% \mathrm{CI}) \\
+\end{array}$} & \multirow{2}{*}{$\begin{array}{l}\text { P-obs } \\
+\end{array}$} & \multicolumn{2}{|c|}{ MAF(\%)* } & \multirow{2}{*}{$\begin{array}{l}\text { OR } \\
(95 \% \mathrm{CI}) \\
\dagger\end{array}$} & \multirow{2}{*}{$\begin{array}{l}\text { P-obs } \\
+\end{array}$} & \multicolumn{2}{|c|}{ MAF(\%)* } & \multirow{2}{*}{$\begin{array}{l}\text { OR } \\
(95 \% \mathrm{CI}) \dagger\end{array}$} & \multirow{2}{*}{$\begin{array}{l}\text { P-obs } \\
*\end{array}$} \\
\hline & & Case & Control & & & Case & Control & & & Case & Control & & & Case & Control & & \\
\hline rs700926 & G & 23.6 & 19.4 & $\begin{array}{ll}1.34 \\
(1.07- \\
1.52) \\
\end{array}$ & $\begin{array}{l}3.2 \mathrm{E}- \\
4\end{array}$ & 26.0 & 19.1 & \begin{tabular}{|l}
1.42 \\
$(1.17-$ \\
$1.59)$ \\
\end{tabular} & $\begin{array}{l}5.4 \mathrm{E}- \\
5\end{array}$ & 24.1 & 19.6 & \begin{tabular}{|l|}
1.40 \\
$(1.08-$ \\
$1.51)$ \\
\end{tabular} & $\begin{array}{l}1.4 \mathrm{E}- \\
5\end{array}$ & 26.3 & 21.4 & $\begin{array}{l}1.25 \\
(0.95-1.44)\end{array}$ & $\begin{array}{l}2.2 \mathrm{E}- \\
4\end{array}$ \\
\hline rs1833529 & G & 22.2 & 19.3 & \begin{tabular}{|l|}
1.30 \\
$(1.15-$ \\
$1.48)$ \\
\end{tabular} & $\begin{array}{l}1.5 \mathrm{E}- \\
4\end{array}$ & 23.4 & 17.9 & \begin{tabular}{|l|}
1.35 \\
$(1.09-$ \\
$1.57)$ \\
\end{tabular} & $\begin{array}{l}1.5 \mathrm{E}- \\
5\end{array}$ & 21.0 & 15.0 & \begin{tabular}{|l|}
1.45 \\
$(1.05-$ \\
$163)$ \\
\end{tabular} & $\begin{array}{l}2.9 \mathrm{E}- \\
5\end{array}$ & 23.7 & 17.5 & $\begin{array}{l}1.38 \\
(1.01-1.45)\end{array}$ & $\begin{array}{l}8.5 \mathrm{E}- \\
4\end{array}$ \\
\hline rs2270915 & G & 20.1 & 14.9 & \begin{tabular}{|l|}
1.43 \\
$(1.12-$ \\
$1.83)$ \\
\end{tabular} & $\begin{array}{l}3.8 \mathrm{E}- \\
3\end{array}$ & 20.9 & 17.6 & \begin{tabular}{|l|}
1.23 \\
$(1.10-$ \\
$152)$ \\
\end{tabular} & 0.05 & 21.0 & 15.0 & \begin{tabular}{|l|}
1.51 \\
$(1.10-$ \\
$1.73)$ \\
\end{tabular} & $\begin{array}{l}1.7 \mathrm{E}- \\
3-\end{array}$ & 21.5 & 16.8 & $\begin{array}{l}1.38 \\
(1.10-1.78)\end{array}$ & $\begin{array}{l}5.1 \mathrm{E}- \\
4\end{array}$ \\
\hline rs17541471 & C & 17.2 & 14.0 & \begin{tabular}{|l|}
1.29 \\
$(1.05-$ \\
$1.61)$ \\
\end{tabular} & 0.02 & 17.5 & 13.4 & \begin{tabular}{|l|}
1.31 \\
$(1.08-$ \\
$1.69)$ \\
\end{tabular} & 0.01 & 17.0 & 13.0 & \begin{tabular}{|l|}
1.30 \\
$(1.03-$ \\
$1.61)$ \\
\end{tabular} & 0.07 & 18.0 & 13.2 & $\begin{array}{l}1.40 \\
(1.05-1.65)\end{array}$ & 0.01 \\
\hline rs3792758 & $\mathrm{T}$ & 17.1 & 13.1 & \begin{tabular}{|l|}
1.32 \\
$(1.10-$ \\
$1.61)$ \\
\end{tabular} & $\begin{array}{l}3.0 \mathrm{E}- \\
3\end{array}$ & 18.4 & 12.8 & \begin{tabular}{|l|}
1.54 \\
$(1.20-$ \\
$1.99)$ \\
\end{tabular} & $\begin{array}{l}7.7 \mathrm{E}- \\
4\end{array}$ & 17.0 & 12.5 & \begin{tabular}{|l|}
1.61 \\
$(1.10-$ \\
$1.82)$ \\
\end{tabular} & $\begin{array}{l}5.1 \mathrm{E}- \\
3\end{array}$ & 17.5 & 13.0 & $\begin{array}{l}1.43 \\
(1.10-1.75)\end{array}$ & $\begin{array}{l}1.0 \mathrm{E}- \\
3\end{array}$ \\
\hline rs696831 & $\mathrm{T}$ & 19.1 & 15.4 & \begin{tabular}{|l|}
1.38 \\
$(1.12-$ \\
$1.75)$ \\
\end{tabular} & 0.02 & 17.3 & 13.7 & \begin{tabular}{|l|}
1.32 \\
$(1.02-$ \\
$1.70)$ \\
\end{tabular} & 0.03 & 18.3 & 15.0 & \begin{tabular}{|l|}
1.40 \\
$(1.06-$ \\
$1.70)$ \\
\end{tabular} & 0.01 & 18.1 & 13.4 & $\begin{array}{l}1.37 \\
(1.12-1.75)\end{array}$ & 0.04 \\
\hline rs7715279 & A & 12.1 & 11.2 & \begin{tabular}{|l|}
1.20 \\
$(0 . .93-$ \\
$1.30)$ \\
\end{tabular} & 0.49 & 11.7 & 9.6 & \begin{tabular}{|l|}
1.25 \\
$(0.94-$ \\
$1.65)$ \\
\end{tabular} & 0.12 & 11.2 & 10.0 & \begin{tabular}{|l|}
1.23 \\
$(0 . .95-$ \\
$1.37)$ \\
\end{tabular} & 0.13 & 12.8 & 12.6 & \begin{tabular}{|l}
0.98 \\
$(0.69-1.40)$
\end{tabular} & 0.93 \\
\hline rs6450922 & G & 25.4 & 24.5 & \begin{tabular}{|l|}
1.25 \\
$(1.02-$ \\
$1.25)$ \\
\end{tabular} & 0.65 & 26.1 & 25.5 & \begin{tabular}{|l|}
1.29 \\
$(1.00-$ \\
$1.67)$ \\
\end{tabular} & 0.05 & 25.3 & 24.9 & \begin{tabular}{|l|}
1.20 \\
$(1.00-$ \\
$1.20)$ \\
\end{tabular} & 0.98 & 251 & 24.6 & $\begin{array}{l}1.18 \\
(0.95-1.20)\end{array}$ & 0.84 \\
\hline rs10941022 & $\mathrm{T}$ & 13.6 & 14.5 & \begin{tabular}{|l|}
0.92 \\
$(0.71-$ \\
$1.21)$ \\
\end{tabular} & 0.57 & 12.7 & 11.6 & \begin{tabular}{|l|}
1.11 \\
$(0.90-$ \\
$1.37)$ \\
\end{tabular} & 0.34 & 14.6 & 13.9 & \begin{tabular}{|l|}
1.10 \\
$(0.98-$ \\
$1.27)$ \\
\end{tabular} & 0.81 & 14.1 & 13.6 & $\begin{array}{l}1.01 \\
(0.98-1.28)\end{array}$ & 0.71 \\
\hline rs976576 & G & 29.2 & 27.0 & \begin{tabular}{|l}
1.00 \\
$(0.85-$ \\
$1.14)$ \\
\end{tabular} & 0.21 & 28.5 & 27.0 & \begin{tabular}{|l}
1.05 \\
$(0.95-$ \\
$1.15)$ \\
\end{tabular} & 0.54 & 28.0 & 27.5 & $\begin{array}{l}1.02 \\
(0.79- \\
1.10) \\
\end{array}$ & 0.69 & 29.3 & 27.1 & $\begin{array}{l}1.12 \\
(0.98-1.21)\end{array}$ & 0.32 \\
\hline
\end{tabular}

* MAF: minor allele frequency. $\dagger \mathrm{OR}$, odds ratio. CI, confidence interval. $\uparrow P$-obs, uncorrected $P$ value.

SNPs were genotyped and analyzed in 596 unrelated Han individuals (293 CAD patients and 293 controls) in Dis-Shanghai cohort. In addition to the known SNPs, we focused on the 25 SNPs representing 7 genomic regions (HFE: rs2071303, rs17596719, rs6918586; CAT: rs769214, rs1049982, rs11032699, rs11032700, rs2179625, rs7104301; HOMX1: rs2071748, rs4820192, rs5755718, rs5755720; CXCL9: rs2276886; PON3: rs978903; LAMA4: rs1016825, rs736160, rs6568719, rs2237248, rs9320402, rs4571602; NPR-C: rs12697273, rs 10066436, rs3828586, rs10061804) using a significant threshold (Supplementary Table S.1). These regions and SNPs were further analyzed from the GWAS scan data with any of the genotype, allele, dominant, and recessive models for subsequent cross-platform validation using Sequenom. The results demonstrated that only 12 SNPs in 3 regions (rs10074149, rs11749133, rs16890187, rs1833530, rs696835 and rs973079 in NPR-C, rs2282854, kgp9060646 and kgp2840023 in LAMA4, rs2074352, rs17884000 and rs17774169 in PON3) showed weak association at stage 1 with the genotype, allele, dominant, or recessive models (Supplementary Table S.2). Furthermore, these $P$-values were below or near the genome-wide significance level of $5^{\prime} 10^{-4}$. In contrast, other 56 SNPs in HMOX1, CXCL9, HFE and CAT genes showed no significant association with CAD by GWAS in Dis-Shandong cohort (Supplementary Table S.2). This discrepancy may result from the limited sample size of our
GWAS, although its population was highly selected from "extreme individuals".

To further test their potential association with CAD in a larger population, 13 tag SNPs (rs2071303 and rs2794719 in HFE; rs554576, rs524154 and rs7947841 in CAT; rs2071749 in HOMX1; rs2276886 and rs2869460 in CXCL9; rs2057682, rs7787187 and rs11977702 in PON3; rs6568719 in LAMA4; rs700926 in NPR-C) that have relatively strong representativeness for these 7 genomic regions, respectively, were selected from the HapMap database and considered for genotyping analysis in the two independent validation populations from Northern (Val-Shandong Cohort) and Southern (Val-Hubei Cohort) China. Except for rs700926 in NPR-C, none of the 12 SNPs being validated (HFE: rs2071303, rs2794719; CAT: rs554576, rs524154, rs7947841; HOMX1: rs2071749; CXCL9: rs2276886, rs2869460; PON3: rs2057682, rs7787187, rs11977702) showed significant association with CAD in Val-Shandong and Val-Hubei cohorts (Supplementary Table S.3), and thus these 12 SNPs did not undergo further analysis.

\section{Novel susceptibility locus for CAD}

Importantly, rs700926 in NPR-C was identified to be consistently associated with CAD in 2 cohorts from Shandong and Hubei populations (Table 2). We further 
Table 3: Allelic association of SNPs of NPR-C with coronary artery disease in 4 combined cohorts (Rep-Shandong, Rep-Hubei, Re-Sichuan and Re-Shaanxi)

\begin{tabular}{|c|c|c|c|c|c|c|c|c|}
\hline \multirow{2}{*}{ SNP_ID } & \multirow{2}{*}{ Position } & \multirow{2}{*}{ Allele } & \multicolumn{2}{|c|}{ MAF(\%) } & \multirow{2}{*}{$p$-HW * } & \multirow{2}{*}{ OR(95\%CI $) \dagger$} & \multirow{2}{*}{ P-obs : } & \multirow{2}{*}{$P$-adj ${ }^{x}$} \\
\hline & & & Case & \begin{tabular}{|l|} 
Control \\
\end{tabular} & & & & \\
\hline rs700926 & 32745283 & $G$ & 24.9 & 19.7 & 0.37 & $1.36(1.17-1.57)$ & $5.0 \mathrm{E}-7$ & $1.2 \mathrm{E}-6$ \\
\hline rs1833529 & 32734140 & $\mathrm{G}$ & 22.9 & 18.5 & 0.45 & \begin{tabular}{|l}
$1.31(1.12-1.52)$ \\
\end{tabular} & $3.0 \mathrm{E}-6$ & $1.0 \mathrm{E}-5$ \\
\hline rs2270915 & 32786389 & G & 20.5 & 16.3 & 0.89 & $1.33(1.13-1.55)$ & $5.7 \mathrm{E}-6$ & $9.0 \mathrm{E}-6$ \\
\hline rs17541471 & 32755589 & $\mathrm{C}$ & 18.4 & 12.8 & 0.33 & \begin{tabular}{|l}
$1.54(1.20-1.99)$ \\
\end{tabular} & $8.2 \mathrm{E}-6$ & $2.0 \mathrm{E}-5$ \\
\hline rs3792758 & 32745752 & $\mathrm{~T}$ & 22.5 & 19.0 & 0.53 & 1.24(1.04-1.47) & 0.014 & 0.026 \\
\hline rs696831 & 32725531 & $\mathrm{~T}$ & 17.1 & 13.7 & 0.67 & $1.30(1.03-1.64)$ & 0.029 & 0.041 \\
\hline rs7715279 & 32689875 & A & 11.8 & 11.0 & 0.78 & $1.08(0.89-1.31)$ & 0.454 & 0.598 \\
\hline rs6450922 & 32689718 & $G$ & 25.3 & 24.8 & 0.82 & $1.29(1.00-1.67)$ & 0.053 & 0.071 \\
\hline rs10941022 & 32691416 & $\mathrm{~T}$ & 12.7 & 11.6 & 0.98 & \begin{tabular}{|l|}
$1.11(0.90-1.37)$ \\
\end{tabular} & 0.343 & 0.542 \\
\hline rs976576 & 32722499 & $\mathrm{G}$ & $36 . .9$ & 37.1 & 0.74 & $0.99(0.86-1.14)$ & 0.752 & 0.915 \\
\hline
\end{tabular}

$* P$-HW, $P$ value for Hardy-Weinberg disequilibrium analysis. $\dagger \mathrm{OR}$, odds ratio, $\mathrm{CI}$, confidence interval. $\$ P$-obs, uncorrected $P$ value. ${ }^{x} P$-adj, $P$ value obtained after adjustment for gender, age, hypertension and diabetes.

analyzed the association between rs700926 and CAD in other 2 cohorts from Northern (Rep-Shaanxi) and Southern (Rep-Sichuan) China and identified a consistent association with CAD, even in combined population from 4 geographically different regions with $P=5.0 \times 10^{-07}$ and $\mathrm{OR}=1.36$ for allele $\mathrm{G}$ (Table 2). To further investigate how the SNP alleles are interacted with traditional risk factors (age, gender, smoking, hypertension and diabetes) in conferring genetic risks for $\mathrm{CAD}$, we conducted genotypic association analysis using multivariate logistic regression analysis. A significant association still existed after adjustment for conventional risk factors for CAD $(P$ adj $\left.=1.2 \times 10^{-6}\right)$ (Table 3$)$. With our sample size and with the alpha level set at 0.05 , there was at least $80 \%$ power for detection of SNPs with OR $\geq 1.3$. These results suggest rs700926 in NPR-C located in Chr 5p confers a highly significant risk of CAD in the northern and southern Chinese Han populations and that rs700926 may represent a new susceptibility locus for CAD.

\section{Association analysis between common variants of NPR-C and CAD}

To assess whether common variants in the $N P R-C$ gene were associated with sporadic CAD in the Chinese population, 9 tag SNPs of NPR-C (rs 1833529, rs2270915, rs 17541471, rs3792758, rs696831, rs7715279, rs6450922, rs 10941022 and $r s 976576)$ were firstly genotyped by the Shandong research group in 4 cohorts from Northern (Rep-Shandong and Rep-Shaanxi) and Southern (Rep-Hubei and Rep-Sichuan) China, respectively (Supplementary Figure S.3). Among these SNPs, 5 tag SNPs $(r s 1833529, r s 2270915, r s 17541471, r s 3792758$ and rs696831) exhibited a significant association with CAD in Rep-Shandong cohort, whereas additional 4 SNPs (rs7715279, rs6450922, rs10941022 and rs976576) showed no significant association with CAD. Then the above 5 positive SNPs were further tested in Rep-Hubei, Rep-Sichuan and Rep-Shaanxi cohorts, respectively, and the results showed positive association with CAD (Table 2 ). Thereafter, we combined the 4 cohorts together and performed further association analysis, which again demonstrated positive association with CAD (Table 3). rs3792758 with larger OR ratios $(1.45(1.10-1.75))$ and lower $p$-values $\left(p=1.0^{-3}\right)$ were observed in Rep-Shaanxi cohorts (Table 2 ), a result induced probably by the small sample size of this cohort. In addition, the Shanghai research group genotyped rs2270915 and additional 10 tag SNPs (rs9716700, rs11750438, rs6889608, rs 10036648, rs12697273, rs10066436, rs3828586, rs2062708, rs 10061804 and rs7730564) in Rep-Center 1 and 2 populations (Supplementary Figure S.4). Of them, only rs12697273 and rs10066436 of NPR-C showed a consistent association with CAD in these two cohorts (Table 4), and these associations remained significant even after adjustment for the statistically significant covariates of age, gender, hypertension, diabetes, smoking, total cholesterol, triglyceride, high-density lipoprotein cholesterol (HDL-C) and low-density lipoprotein cholesterol (LDL-C). Four SNPs of them (rs2270915, rs10036648, rs10061804 and rs3828586) exhibited a positive association with CAD only in Rep-Center 1 but not in Rep-Center 2 populations. Furthermore, there is no mutation of rs9716700 in the promoter region of the $N P R-C$ gene in the central China population. In each of the 4 cohorts, the remaining 4 SNPs in introns (rs11750438, rs6889608, rs7730564 and rs2062708) showed no significant association with CAD. Though rs700926 has been identified to be associated with CAD in the northern and southern China population, rs10061804 and rs3828586, which are well representative of rs700926 (both $r^{2}=1.0$ ) (Figure 2), did not show similar association in the two central China cohorts. According to HardyWeinberg equilibrium analysis, rs700926 is also well 
Table 4: Allelic association of SNPs of NPR-C with coronary artery disease in Central Chinese Han population

\begin{tabular}{|c|c|c|c|c|c|c|c|c|}
\hline \multirow{2}{*}{ Population } & \multirow{2}{*}{ SNP_ID } & \multirow{2}{*}{ Position } & \multicolumn{2}{|c|}{ MAF(\%)* } & \multirow{2}{*}{ Allele } & \multirow{2}{*}{$\mathrm{OR}(95 \% \mathrm{CI}) \dagger$} & \multirow[t]{2}{*}{ P-obst: } & \multirow[t]{2}{*}{$P$-adj ${ }^{X}$} \\
\hline & & & case & control & & & & \\
\hline \multirow{11}{*}{ Cohort 1} & rs9716700 & 32711633 & 0 & 0 & A & & 1 & \\
\hline & rs11750438 & 32716535 & 0.143357 & 0.121993 & $\mathrm{~T}$ & $0.83(0.59-1.17)$ & 0.297177 & \\
\hline & rs6889608 & 32719693 & 0.305263 & 0.261168 & $\mathrm{~T}$ & $0.80(0.62-1.04)$ & 0.0734387 & \\
\hline & rs 10036648 & 32724078 & 0.166084 & 0.146048 & G & $1.16(0.85-1.60)$ & 0.040828 & \\
\hline & rs12697273/10037355 & 32730464 & 0.258741 & 0.185567 & $\mathrm{~T}$ & $0.65(0.49-0.86)$ & 0.0088942 & \\
\hline & rs10066436 & 32740346 & 0.255245 & 0.183849 & $\mathrm{~T}$ & $0.66(0.50-0.87)$ & 0.0111536 & \\
\hline & rs 3828586 & 32746547 & 0.265734 & 0.197595 & G & $1.47(1.12-1.94)$ & 0.021309 & \\
\hline & rs10061804 & 32748637 & 0.265734 & 0.197595 & A & $0.68(0.52-0.90)$ & 0.021309 & \\
\hline & rs2062708 & 32753672 & 0.185315 & 0.146048 & $\mathrm{C}$ & $1.33(0.97-1.82)$ & 0.142288 & \\
\hline & rs 7730564 & 32767727 & 0.185315 & 0.14433 & G & $1.35(0.99-1.84)$ & 0.118293 & \\
\hline & rs2270915 & 32786389 & 0.20979 & 0.185567 & $G$ & $1.17(0.87-1.56)$ & 0.0460445 & \\
\hline \multirow{7}{*}{ Cohort 2} & rs9716700 & 32711633 & 0 & 0.000661 & A & & 0.424719 & \\
\hline & rs10036648 & 32724078 & 0.171329 & 0.155378 & G & $1.12(0.94-1.35)$ & 0.290962 & \\
\hline & rs $12697273 / 10037355$ & 32730464 & 0.1865 & 0.220961 & $\mathrm{~T}$ & $1.24(1.05-1.46)$ & 0.0107192 & \\
\hline & rs 10066436 & 32740346 & 0.182548 & 0.22237 & $\mathrm{~T}$ & $1.28(1.08-1.51)$ & 0.00484991 & \\
\hline & rs 3828586 & 32746547 & 0.202663 & 0.236037 & G & $0.82(0.70-0.97)$ & 0.0592019 & \\
\hline & rs10061804 & 32748637 & 0.203156 & 0.225033 & A & $1.14(0.97-1.34)$ & 0.295754 & \\
\hline & rs2270915 & 32786389 & 0.174729 & 0.181941 & G & $0.95(0.80-1.13)$ & 0.688235 & \\
\hline \multirow{2}{*}{ Combined } & rs $12697273 / 10037355$ & 32730464 & & & & & & $1.2 \mathrm{E}-3$ \\
\hline & rs10066436 & 32740346 & & & & & & $1.5 \mathrm{E}-4$ \\
\hline
\end{tabular}

* MAF: minor allele frequency. $\dagger$ OR, odds ratio. CI, confidence interval. $\$ P$-obs, uncorrected $P$ value. ${ }^{\mathrm{X}} P$-adj, $\mathrm{P}$ value obtained after adjustment for gender, age, hypertension, and diabetes.

Table 5: Allelic association of $r s 12697273$ and $r s 10066436$ with coronary artery disease in Rep-Shandong and RepHubei cohorts

\begin{tabular}{|c|c|c|c|c|c|c|}
\hline \multirow{2}{*}{ SNP_ID } & \multirow{2}{*}{ Population } & \multicolumn{2}{|l|}{ MAF(\%)* } & \multirow{2}{*}{ Allele } & \multirow{2}{*}{ OR(95\%CI) † } & \multirow[t]{2}{*}{$P$-obs +} \\
\hline & & case & control & & & \\
\hline \multirow{3}{*}{ rs 12697273} & Rep-Shandong & 0.17314 & 0.196203 & $\mathrm{~T}$ & $0.86(0.64-1.15)$ & 0.3111427 \\
\hline & Rep-Wuhan & 0.209239 & 0.188716 & $\mathrm{~T}$ & $1.14(0.90-1.14)$ & 0.28547 \\
\hline & Combined & 0.18559 & 0.190476 & $\mathrm{~T}$ & $0.94(0.80-1.11)$ & 0.48005526 \\
\hline \multirow{3}{*}{ rs10066436 } & Rep-Shandong & 0.19237 & 0.201721 & $\mathrm{~T}$ & $1.07(0.80-1.41)$ & 0.375217 \\
\hline & Rep-Wuhan & 0.213043 & 0.192308 & $\mathrm{~T}$ & $1.14(0.85-1.52)$ & 0.387946891 \\
\hline & Combined & 0.201325 & 0.193023 & $\mathrm{~T}$ & $1.10(0.83-1.40)$ & 0.573591 \\
\hline
\end{tabular}

$*$ MAF: minor allele frequency. $\uparrow \mathrm{OR}$, odds ratio. CI, confidence interval. $\ddagger P$-obs, uncorrected $P$ value.

representative of rs1833529, rs17541471 and rs3792758 (Figure 2). Thereafter, the two new susceptibility loci (rs12697273 and rs10066436) identified in the central China population were further replicated in Rep-Shandong and Rep-Hubei cohorts, and the result showed no significant association between these two SNPs and CAD (Table 5). In addition, we performed association analysis between the remaining SNPs not associated with CAD and the known risk factors for CAD and did not find any significant association between them (Table 3 ).

\section{Association of the risk allele $G$ of rs700926 with expression of NPR-C mRNA in human peripheral blood leukocytes}

Because these positively associated SNPs (rs700926, rs 1833529, rs2270915, rs 17541471, rs3792758, rs696831, rs12697273 and rs10066436) are not located in the promoter or extron of NPR-C, they may not influence directly the expression levels of NPR-C mRNA. Compared with other seven SNPs, rs700926 is located near intron 1 of NPR-C, and thus we hypothesized 
that it may affect the expression levels of the NPR-C mRNA. To test this hypothesis, we used real-time PCR analysis to measure the NPR-C expression levels in human peripheral blood leukocytes from 95 individuals with different genotypes. As shown in Figure $3 \mathrm{~A}$, the mRNA expression levels of NPR-C were significantly higher in 63 individuals with the TT genotype than in 32 individuals with the GG or GT genotype. To further confirm these results, we performed a replication study with a randomly selected group of 380 individuals. As shown in Figure 3B, the mRNA expression level of NPR-C was also significantly higher in 226 individuals with the TT genotype than in 254 individuals with the GG or GT genotype.

\section{DISCUSSION}

In the present study, we performed a multicenter GWAS, pathway-based candidate gene association and replication studies in 6 geographically different Chinese Han populations and identified new susceptibility loci of $N P R-C$ that was specifically associated with CAD.

In the first stage of the study, we found sufficient evidence for a potentially novel SNP rs700926 in NPR-C gene associated with CAD in Han Chinese. Then we identified other 5 tag SNPs (rs1833529, rs2270915, rs 17541471, rs3792758 and rs696831) associated with CAD simultaneously in the northern and southern Han Chinese population. In addition, another 2 novel

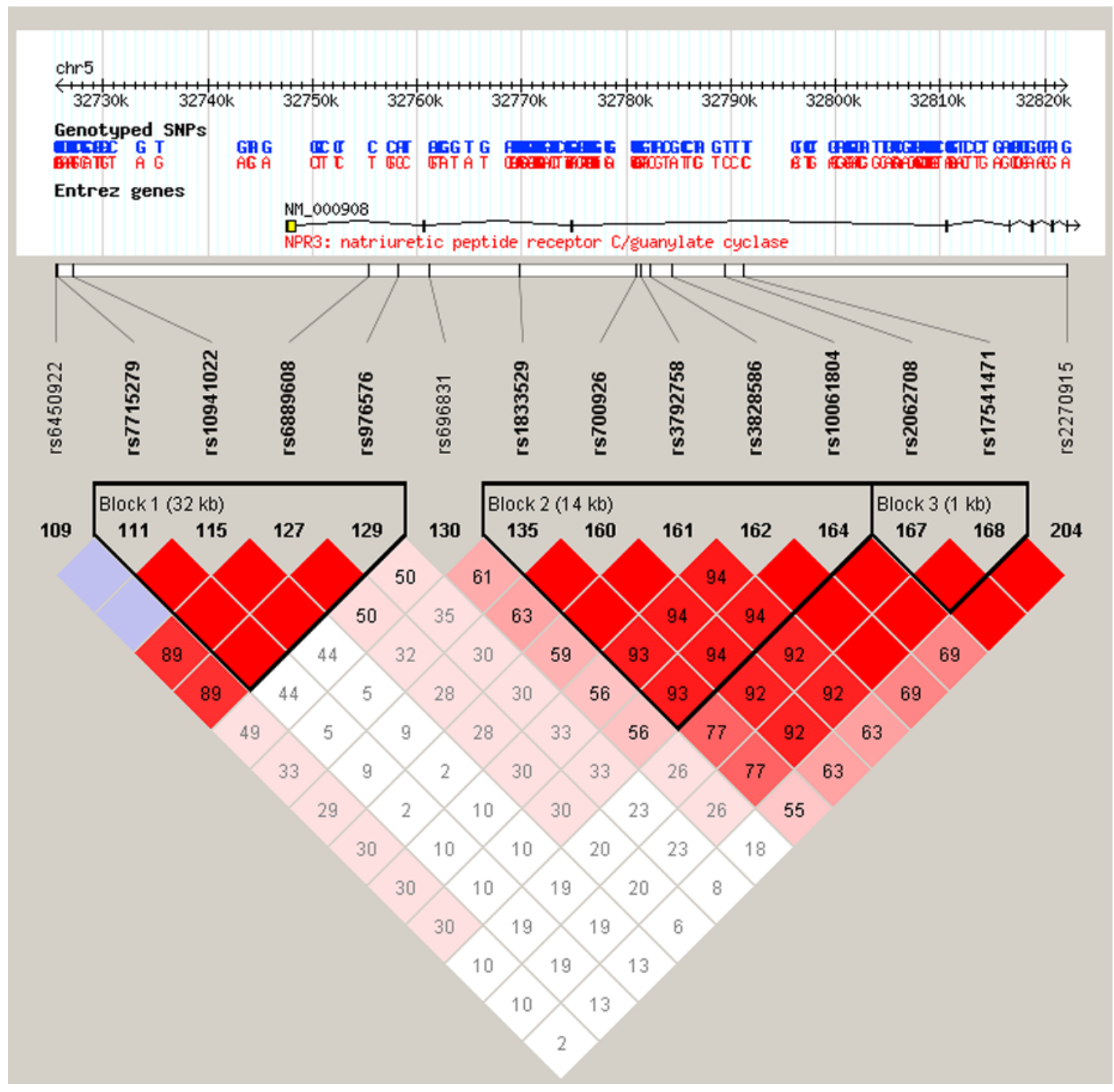

Figure 2: Block structure of linkage disequilibrium for the tag SNPs in the natriuretic peptide receptor $\mathrm{C}$ gene region (NPR-C) on chromosome 5p13.3. The linkage disequilibrium (LD) of the tag SNPs with other SNPs were assessed respectively using the data from HapMap by a D'> 0.9 and an $\mathrm{r}^{2}>0.7$ as standard. Note that rs700926 has relatively strong representativeness of rs 1833529 , rs17541471, rs3792758 and rs3828586. 
tag SNPs (rs12697273 and rs10066436) in NPR-C gene also showed significant association with CAD in the central Han Chinese. Noticeably, these significant associations remained significant even after adjustment for conventional risk factors of atherosclerosis (age, gender, smoking, hypertension, diabetes and hyperlipidemia), suggesting that the contribution of these polymorphisms to the risk of CAD is independent of conventional risk factors. In addition to the strict criteria used for defining CAD and normal phenotypes in this study, all CAD patients and normal controls were verified to be genetically unrelated by examining their familial relationships. In order to further validate these findings, we recruited a large number of subjects from northern, southern and central China, who were well representative of the general Han Chinese population [10]. Importantly, crossreplication of the results from 5 independent populations may decrease the rate of false positives. Therefore, our results are unlikely due to a specific cohort effect and are able to identify significant SNPs associated with CAD despite the small sample size in the discovery study.

As the largest ethnic group in mainland China, Han Chinese is a geographically and genetically heterogeneous population and has subpopulation structures, which may have considerable effect on the design and interpretation of replication studies [10]. In the present study, we identified 6 associated SNPs of NPR-C (rs700926, rs 1833529 , rs2270915, rs 17541471, rs3792758 and rs696831) in the northern and southern Chinese Han population and 2 different associated SNPs (rs 12697273 and rs 10066436) of NPR-C in the central Chinese Han population. As rs3828586, rs 10061804 and rs700926 have strong LD association with each other, $r s 700926$ is well representative of rs3828586 and rs10061804. Although rs700926 and rs2270915 have been verified to be associated with the risk of CAD in the northern and southern Han Chinese populations, rs3828586, rs 10061804 and rs2270915 didn't show a consistent association with CAD in the central Han Chinese population. Similarly, another two CAD-associated SNPs (rs12697273 and rs 10066436) found in the central Chinese Han population did not show a consistent association with CAD in the northern and southern Han Chinese. These results further demonstrated the diversity of the Chinese human genome. Furthermore, allele effects in the central Han Chinese were consistently weaker than those in the northern and southern Han

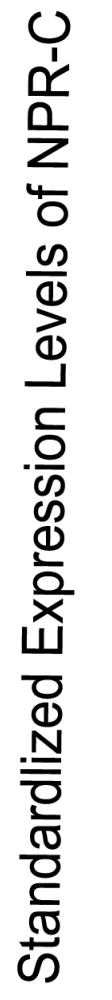

A

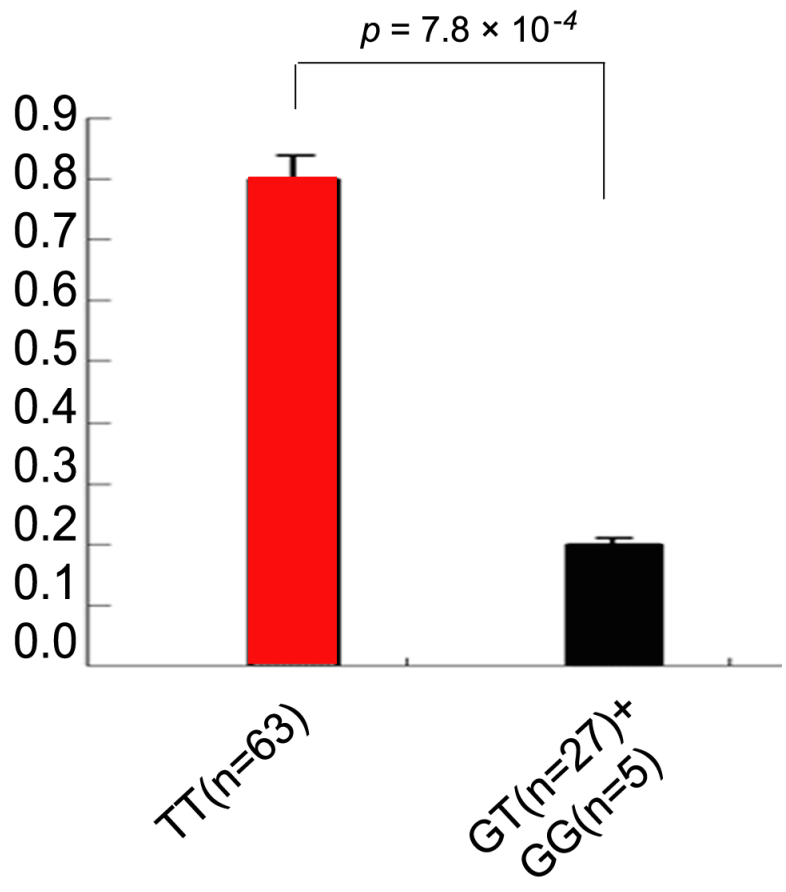

B

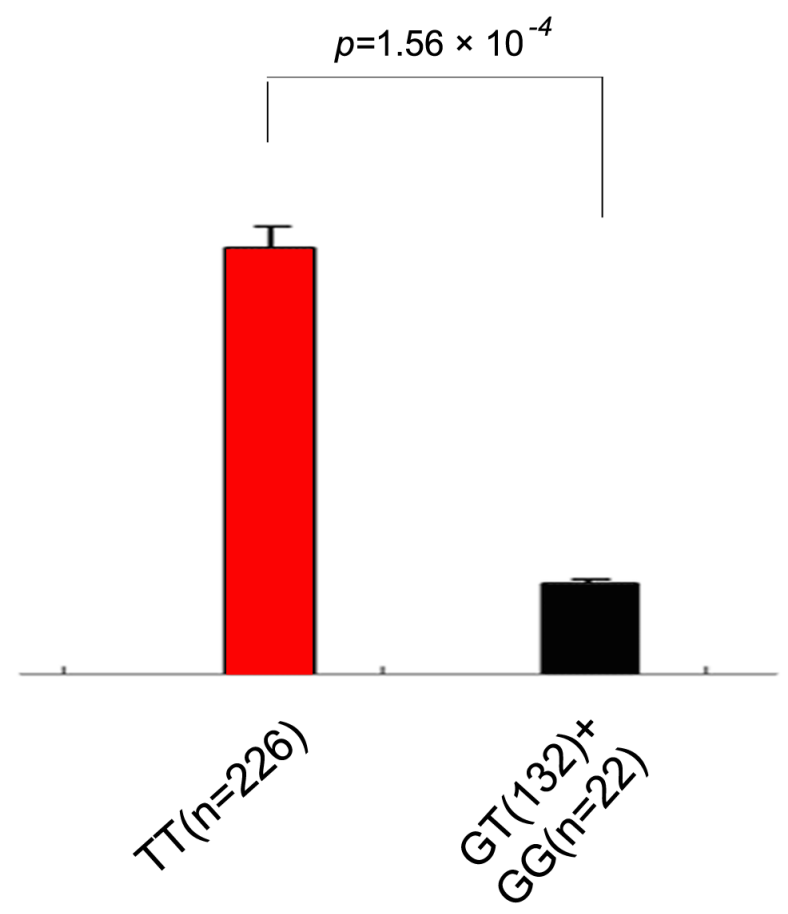

Figure 3: Association of the risk allele G of rs700926 with decreased expression of NPR-C mRNA assuming a dominant model. Because rs700926 is located near intron 1 of NPR-C, we hypothesized that it may affect the expression levels of the NPR-C mRNA. A. 5 individuals with a minor genotype of GG, 27 individuals with genotype GT and 63 individuals with genotype TT of rs 700926 were selected for RT-PCR analysis. B. To validate the finding of a quantitative expression trait locus from the initial analysis, a replication study was also performed in an independent population of 380 randomly selected individuals from the replication populations (northern and southern Chinese). 
Chinese, suggesting that there is a greater genetic difference between central and northern or southern Chinese populations. In the prior GWAS for CAD in Chinese population, genome-wide significant associations of loci in or near C6orf105, TTC32-WDR35, GUCY1A3, C6orf10-BTNL2 and ATP2B1 with CAD were reported by Wang $\mathrm{F}$ et al and Lu XF et al independently [11, 12]. However, the new loci identified by Wang $\mathrm{F}$ et al were not confirmed by Lu XF et al. Similarly, these new loci were not verified in our cohorts. These inconsistent results may be due to different genetic backgrounds in geographically different Chinese Han population. In the study of Wang $\mathrm{F}$ et al, the subjects were recruited from the northern and central China, whereas in the study of Lu XF et al, all participants in the discovery stage and the majority of cases and controls in the replication stage were enrolled from the northern China. By comparison, our subjects were recruited from the northern, southern and central Han Chinese who is well representative of the general Han Chinese population. In addition, normal controls were defined only by normal history and physical examination in previous studies, whereas in our study, only those with normal history, physical examination, blood chemistry and angiography were included as normal controls. Thus, the strict criteria for defining CAD patients and normal controls may explain in part the different results between our and previous studies.

The NPR-C gene resides on human chromosome 5p14-p13, and $N P R-C$ represents the most widely and abundantly expressed natriuretic peptide receptor (NPR), with a tissue distribution that includes most of the major endocrine glands, lungs, kidneys and vessels [13]. In particular, NPR-C is the most common type of NPR in endothelial cells [14]. Although NPR-C was traditionally regarded as a clearance receptor of natriuretic peptides (NPs), recent studies have revealed multiple effects of $N P R-C$ on different cells and organs $[14,15]$ and most of these effects were enhanced by $\mathrm{C}$-type natriuretic peptide (CNP) stimulation rather than by A-type atrial natriuretic peptide (ANP) and B-type natriuretic peptide (BNP) [13]. Recently, a study in 200,000 European descents also showed an association between rs1173771 in NPR3-C5orf23 with hypertension [16]. However, the association between $N P R-C$ gene and CAD has never been reported in any population studies. In the current study, 6 SNPs of NPR-C (rs700926, rs 1833529, rs2270915, rs 17541471, rs3792758 and rs696831) were identified to be associated with CAD in the northern and southern Chinese populations and another two SNPs (rs12697273 and $r s 10066436$ ) found to be associated with CAD in the central Chinese population. Moreover, multivariate logistical regression analysis showed that the association between these SNPs and CAD remained significant even after adjustment for conventional risk factors of CAD including hypertension.

The molecular mechanisms underlying the association between NPR-C gene polymorphism and CAD are unclear but preliminary results revealed a potential role of $N P R-C$ in the pathogenesis of atherosclerosis. A recent study showed that $N P R-C$ expression was increased in neointimal smooth muscle cells in patients receiving percutaneous coronary intervention [17]. Molecular imaging technique demonstrated the presence of NPR-C near the luminal surface of atherosclerotic plaques and in VSMCs [18]. Moreover, NPR-C-dependent ERK $1 / 2$ phosphorylation activated the vasoprotective effect of CNP, resulting in augmentation of endothelial cell proliferation and inhibition of VSMCs growth [19]. Furthermore, Fox AA et al found that 4 NPR-C SNPs (rs700923, rs 16890196, rs765199, rs700926) were associated with left ventricular dysfunction after coronary artery bypass grafting and able to predict patient outcome when combined with NPRC SNPs [20]. In this study, we also detected NPR-C expression in leukocytes from individuals with different genotypes. Although rs 700926 near intron 1 of NPR-C may not directly influence the expression levels of the NPR-C mRNA, we found that the mRNA expression level of NPR-C in human peripheral blood leukocytes was significantly higher in individuals with the TT genotype than those with the GG or GT genotype. These findings are consistent with established effects of NPR-C variant on CAD.

We also investigated the association of some conflicting SNPs (HFE: rs2071303, rs2794719; CAT: rs554576, rs524154, rs7947841; HOMX1: rs2071749; CXCL9: rs2276886, rs2869460; PON3: rs2057682, rs7787187, rs 11977702; LAMA4: rs6568719) with CAD in our Chinese populations. Recently, a case-control study from Hubei province of China found that SNP rs9366637 in HFE gene was associated with higher CAD risk in their study populations [21]. Although our study found a significant association between SNP rs2794719 in $H F E$ gene and CAD from Hubei cohort, this association was not confirmed in the southern (Rep-Sichuan) and northern (Rep-Shandong and Rep-Shaanxi) Han Chinese populations. Furthermore, SNP rs2071303 in HFE gene showed no significant association with CAD in these cohorts. Previous studies investigated the role of the polymorphisms of Heme oxygenase-1 (HMOX1), the only inducible form of heme-oxygenases, in the development of CAD. However, the results of these studies were conflicting $[22,23]$. Similar to the result of Lin $\mathrm{R}$ et al [24], our study in the southern and northern Han Chinese populations showed insignificant association between SNP rs2071749 in HMOX-1 gene and CAD. Experimental studies also showed that paraoxonases (PONs) including PON1, PON2 and PON3 may prevent low density lipoprotein cholesterol (LDL-C) from peroxidation and play a protective role in atherogenesis [25]. Rull A et al [26]. reported a positive association between serum PON3 and $\beta$-2-microglobulin, CCL2 and high-sensitivity C-reactive protein in CAD patients but insignificant 
differences in PON3 gene promoter polymorphisms and their haplotypes between CAD patients and controls, indicating that these associations were not genetically determined. We also genotyped 3 tag SNPs ( $r s 2057682$, rs7787187 and rs 11977702) in PON3 gene and identified no association between these SNPs and CAD.

There are several limitations of our study that must be acknowledged. First, the sample size in the discovery stage was small. However, the sample size in our validation and replication stages was large enough to rule out the possibility that our findings of $N P R-C$ as a novel susceptibility locus of CAD in the Chinese Han population were false positive. Second, some positive SNPs identified in some cohorts of the current study became negative in other cohorts. A possible explanation is that different populations may have different modifier genes, lifestyles, or gene-gene and gene-environment interactions. Third, although the association between $N P R-C$ polymorphism and CAD was highly significant in our study population, other variants in nearby genes in strict linkage disequilibrium with $N P R-C$ polymorphism may be responsible for the observed genetic association. It is likely that the positive SNPs identified in this study may be a simple marker for CAD whereas the true causative SNP in LD with these positive SNPs at 5p13 may be located at a considerably large distance from these SNPs. Fourth, because it was difficult to obtain sufficient human coronary artery specimens, the association of the risk allele of $r s 700926$ with expression of NPR-C mRNA in human coronary artery was not investigated in this study. Finally, our findings are limited to Chinese Han population and further studies are required to confirm the role of $N P R-C$ as a novel susceptibility locus of CAD in other Asian or Western populations.

In conclusion, our study showed 8 SNPs including rs700926, rs 1833529, rs2270915, rs17541471, rs3792758, rs696831, rs 12697273 and rs10066436 in NPR-C gene on chromosome $5 \mathrm{p} 13$ are associated with increased susceptibility to CAD in Chinese Han population. This finding may lead to the discovery of novel pathways in atherogenesis and hence, new preventive and therapeutic targets in CAD patients. Further studies are warranted to identify the true causative variant(s) for CAD at this new 5p13 CAD locus.

\section{MATERIALS AND METHODS}

\section{Power analysis}

Before implementation of this study, we performed a statistical power analysis using the PS version 3.0.43 to ascertain whether the recruited samples could provide adequate power for identifying the association between the modest-effect-size SNPs and CAD, provided that the NPR-C SNPs locus confers the same size of risk for development of CAD in Chinese Han population. Under the population parameter settings of the effect size of odd ratios of 1.30 and the allelic frequency of 0.16 chiefly derived from Hapmap database, and on the basis of our preliminary experiment, our samples with 3363 wellcharacterized CAD cases and 3148 healthy controls can provide a statistical power of $98.2 \%$ and $92.9 \%$ at the nominal Type I error rate of 0.05 and 0.01 , respectively. The power analysis indicates that our Han Chinese sample size is sufficient for identifying these new susceptible loci.

\section{Study populations}

A flow chart of the study protocol was provided in Figure 1. A three-stage case-control study was designed to evaluate the association between genetic variants across human genome and the risk of CAD. To avoid the potential confounding ethnic factors and minimize subpopulation stratification, cases and controls in any cohorts were recruited from the same geographical region. In stage 1 study, the "extreme cases" were defined as CAD patients with no more than one conventional risk factor. The "extreme controls" were defined as subjects with more than three risk factors but without visible coronary arterial narrowing by coronary angiography or CTA. In stage 2 and 3 studies, the subjects with $>50 \%$ coronary stenosis in at least one main vessel identified by selective coronary angiography or computed tomography angiography (CTA) and those who had myocardial infarction and/or underwent percutaneous coronary intervention or coronary artery bypass graft, were classified as CAD cases. Only subjects without any history of cardiovascular diseases and without any visible coronary stenosis by coronary angiography or CTA were included as controls. The study protocol conformed to the principles of the Declaration of Helsinki and was approved by the local Ethics Committee in each hospital involving in this project. A written informed consent was given by all participants before enrollment.

\section{DNA extraction}

Genomic DNA was extracted from EDTAanticoagulated peripheral whole blood using the Wizard genomic DNA purification kit (Promega, Madison, MA, USA) following standard laboratory protocols.

\section{GWAS}

In the discovery stage, genotyping was carried out in 200 "extreme individuals" (Table 1) using Infinium HumanOmnizhonghua-8 BeadChip (Illumina). Highquality genotyping was performed by a commercial company (Bioassay Laboratory of CapitalBio Corporation, 
Changping District, Beijing, China). Before association analysis, quality control call rates and genotyping calls for each array were analyzed by Illumina Genotyping Console software using the Dynamic Model and BRLMM-P algorithms, respectively.

\section{Pathway-based candidate gene case-control association study}

Because of the relatively small sample size of the current GWAS, a candidate gene association study was simultaneously carried out based on known signal pathways for CAD. Based on the primary results of GWAS for CAD in the 200 "extreme individuals" in combination with earlier reports of the highthroughput dataset from a British population by WTCCC (http://www.wtccc.org. uk/), we selected 120 SNPs located in 17 chromosome regions (Chr.1, 2, 3, 4, 5, 6, 7, 8, 9, 10, 11, 12, 16, 18, 19, 20 and 22) to examine their potential associations with CAD in 293 CAD cases and sex/age matched 293 controls in Shanghai (Supplementary Table S.1).

\section{SNP selection and genotyping in the replication study}

Only a fraction of the genotypes from the WTCCC and variants from GWAS were selected for stage 2 validation studies based on the following strict criteria: (1) SNPs had $P \leq 10^{-4}$ for all GWAS samples; (2) SNPs showed consistent associations in pathway-based candidate gene case-control association study at $P \leq 10$ 2; (3) SNPs were not located in the same chromosome regions as reported in previous GWAS; (4) SNPs had clear genotyping clusters; (5) Only the SNP with the lowest $P$ value was selected when multiple SNPs were observed in a strong linkage disequilibrium (LD) $\left(\mathrm{r}^{2} \geq 0.8\right)$; (6) SNPs that had been previously reported to be strongly associated with CAD in Chinese Han population were excluded. A total of 13 SNPs representative of 7 genomic regions were selected for stage 2 validation studies and only SNPs significantly associated with CAD in stage 2 validation studies were selected for stage 3 replication studies. To determine whether common variants of $N P R-C$ gene might be associated with CAD, $r s 2270915$ and nineteen tag SNPs were selected with a $\mathrm{D}^{\prime}>0.9$ and a $\mathrm{r}^{2}>0.7$ as a standard from the HapMap database and genotyped. In the candidate gene association study and at the validation and replication stages, genotyping was carried out with TaqMan technology (Applied Biosystems, Foster City, CA) as described previously.

\section{Quantitative reverse transcription polymerase chain reaction}

Quantitative reverse transcription polymerase chain reaction (qRT-PCR) was performed to determine the mRNA expression of NPR-C in human peripheral blood leukocytes.

\section{Linkage disequilibrium (LD) and statistical genetics}

Statistical analysis for GWAS was performed using previously described methods $[5,6]$. With SPSS for Windows version 13.0 (SPSS Inc., Chicago, IL, USA) and PLINK software, multivariate logistic regression analysis was performed to test whether the association between a SNP and CAD remained significant after adjusting for significant risk factors for CAD. In the replication study, $P<0.05$ was deemed as statistically significant.

\section{ACKNOWLEDGMENTS}

We thank Ma L, An GP, Chen WQ, Kan XH, Jiang $\mathrm{H}$, Qu ZH, Huang SY and Zhang W for their technical assistance. We also thank the staff of Bioassay Laboratory of CapitalBio Corporation for their chip-detection services.

\section{CONFLICTS OF INTEREST}

The authors declare no competing financial interests.

\section{GRANT SUPPORT}

This work was supported by the National 973 Basic Research Program of China (No 2012CB518603, No 2013CB530703, No 2015CB553604), the Program of Introducing Talents of Discipline to Universities (No. B07035), the State Program of National Natural Science Foundation of China for Innovative Research Group (No. 81321061), the State Key Program of National Natural Science of China (No. 61331001, No.81530014), the International Collaboration and Exchange Program of China (No. 81320108004) and the grants of the National Natural Science Foundation of China (No. 81100204, $81370410,81425004,91339109,81100207,81173251$, $81100102,81270350,81000126,81000127)$.

\section{REFERENCES}

1. Mathers CD, Loncar D. Projections of global mortality and burden of disease from 2002 to 2030. PLoS Med. 2006; 3:e442. doi: 10.1371/journal.pmed.0030442

2. Sing CF, Stengard JH, Kardia SLGenes, environment, and 
cardiovascular disease. Arterioscler Thromb Vasc Biol. 2003; 23:1190-1196.

3. Hirschhorn JN, Daly MJ. Genome-wide association studies for common diseases and complex traits. Nat Rev Genet. 2005; 6:95-108.

4. McPherson R, Pertsemlidis A, Kavaslar N, Stewart A, Roberts R, Cox DR, Hinds DA, Pennacchio LA, TybjaergHansen A, Folsom AR, Boerwinkle E, Hobbs HH, Cohen JC.A common allele on chromosome 9 associated with coronary heart disease. Science. 2007; 316:1488-1491.

5. Samani NJ, Erdmann J, Hall AS, Hengstenberg C, Mangino M, Mayer B, Dixon RJ, Meitinger T, Braund P, Wichmann HE, Barrett JH, König IR, Stevens SE, et al. WTCCC and the Cardiogenics Consortium. Genomewide association analysis of coronary artery disease. N Engl J Med. 2007; 357:443-453.

6. Willer CJ, Sanna S, Jackson AU, Scuteri A, Bonnycastle LL, Clarke R, Heath SC, Timpson NJ, Najjar SS, Stringham HM, Strait J, Duren WL, Maschio A, et al. Newly identified loci that influence lipid concentrations and risk of coronary artery disease. Nat Genet. 2008; 40:161-169.

7. Wellcome Trust Case Control Consortium. Genome-wide association study of 14,000 cases of seven common diseases and 3,000 shared controls. Nature. 2007; 447:661-678.

8. Trégouët DA, König IR, Erdmann J, Munteanu A, Braund PS, Hall AS, Grosshennig A, Linsel-Nitschke P, Perret C, DeSuremain M, Meitinger T, Wright BJ, Preuss M, Balmforth AJ, et al. Genome-wide haplotype association study identifies the SLC22A3-LPAL2-LPA gene cluster as a risk locus for coronary artery disease. Nat Genet. 2009; 41:283-285.

9. Myocardial Infarction Genetics Consortium1, Kathiresan S, Voight BF, Purcell S, Musunuru K, Ardissino D, Mannucci PM, Anand S, Engert JC, Samani NJ,Schunkert H, Erdmann J, Reilly MP, et al. Genome-wide association of early-onset myocardial infarction with single nucleotide polymorphisms and copy number variants. Nat Genet. 2009; 41:334-341.

10. Chen J, Zheng H, Bei JX, Sun L, Jia WH, Li T, Zhang F, Seielstad M, Zeng YX, Zhang X, Liu J. Genetic structure of the Han Chinese population revealed by genome-wide SNP variation. Am J Hum Genet. 2009; 85:775-785.

11. Wang F, Xu CQ, He Q, Cai JP, Li XC, Wang D, Xiong X, Liao YH, Zeng QT, Yang YZ, Cheng X, Li C, Yang R, et al. Genome-wide association identifies a susceptibility locus for coronary artery disease in the Chinese Hanpopulation. Nat Genet. 2011; 43:345-349.

12. Lu X, Wang L, Chen S, He L, Yang X, Shi Y, Cheng J, Zhang L, Gu CC, Huang J, Wu T, Ma Y, Li J, et al. Genome-wide association study in Han Chinese identifies four new susceptibility loci for coronary artery disease. Nat Genet. 2012; 44:890-894.

13. Rose RA, Giles WR. Natriuretic peptide C receptor signalling in the heart and vasculature. J Physiol 2008; 586:353-366.
14. Leitman DC, Andresen JW, Kuno T, Kamisaki Y, Chang JK, Murad F. Identification of multiple binding sites for atrial natriuretic factor by affinity cross-linking in cultured endothelial cells. J Biol Chem. 1986; 261:11650-11655.

15. Maack T, Suzuki M, Almeida FA, Nussenzveig D, Scarborough RM, McEnroe GA, Lewicki JA. Physiological role of silent receptors of atrial natriuretic factor. Science. 1987; 238:675-678.

16. International Consortium for Blood Pressure Genome-Wide Association Studies, Ehret GB, Munroe PB, Rice KM, Bochud M, Johnson AD, Chasman DI, Smith AV, Tobin MD, Verwoert GC, Hwang SJ, Pihur V, Vollenweider P, et al. Genetic variants in novel pathways influence blood pressure and cardiovascular disease risk. Nature. 2011; 478:103-109.

17. Naruko T, Itoh A, Haze K, Ehara S, Fukushima H, Sugama Y, Shirai N, Ikura Y, Ohsawa M, Ueda M. C-type natriuretic peptide and natriuretic peptide receptors are expressed by smooth muscle cells in the neointima after percutaneous coronary intervention. Atherosclerosis. 2005; 181:241-250.

18. Liu Y, Abendschein D, Woodard GE, Rossin R, McCommis K, Zheng J, Welch MJ, Woodard PK. Molecular imaging of atherosclerotic plaque with (64)Cu-labeled natriuretic peptide and PET. J Nucl Med. 2010; 51:85-91.

19. Khambata RS, Panayiotou CM, Hobbs AJ Natriuretic peptide receptor-3 underpins the disparate regulation of endothelial and vascular smooth muscle cell proliferation by C-type natriuretic peptide. Br J Pharmacol. 2011; 164:584597.

20. Fox AA, Collard CD, Shernan SK, Seidman CE, Seidman JG, Liu KY, Muehlschlegel JD, Perry TE, Aranki SF, Lange C, Herman DS, Meitinger T, Lichtner P,Body SC. Natriuretic peptide system gene variants are associated with ventricular dysfunction after coronary artery bypass grafting. Anesthesiology. 2009; 110:738-747.

21. Shi Y, Zhou L, Huang LH, Lian YT, Zhang XM, Guo $\mathrm{H}, \mathrm{Wu} \mathrm{TC}$, Cheng LX, He MA. Plasma ferritin levels, genetic variations in HFE gene, and coronary heart disease in Chinese: a case-control study. Atherosclerosis. 2011; 218:386-390.

22. Qiao H, Sai X, Gai L, Huang G, Chen X, Tu X, Ding Z. Association between heme oxygenase 1 gene promoter polymorphisms and susceptibility to coronary artery disease: a HuGE review and meta-analysis. Am J Epidemiol. 2014; 179:1039-1048.

23. Lin R, Wang Y, Wang Y, Fu W, Zhang D, Zheng H, Yu T, Wang Y, Shen M, Lei R, Wu H, Sun A, Zhang R, Wang $\mathrm{X}$, Xiong M, Huang W, Jin L. Common variants of four bilirubin metabolism genes and their association with serum bilirubin and coronary artery disease in Chinese Han population. Pharmacogenet Genomics. 2009; 19:310-318.

24. Draganov DI, Teiber JF, Speelman A, Osawa Y, Sunahara $\mathrm{R}$, La Du BN. Human paraoxonases (PON1, PON2, and PON3) are lactonases with overlapping and distinct 
substrate specificities. J Lipid Res. 2005; 46:1239-1247.

25. Rull A, García R, Fernández-Sender L, García-Heredia A, Aragonès G, Beltrán-Debón R, Marsillach J, Alegret JM, Martín-Paredero V, Mackness B, Mackness M, Joven J, Camps J. Serum paraoxonase-3 concentration is associated with insulin sensitivity in peripheral artery disease and with inflammation in coronary artery disease. Atherosclerosis. 2012; 220:545-551.
26. Shen GQ, Abdullah KG, Wang QK. The TaqMan method for SNP genotyping. Methods Mol Biol. 2009; 578:293306. 